\title{
Meta-patrones Morfogenéticos: Propuesta de Framework para Arquitecturas Generativas Basadas en Desempeño
}

\author{
Morphogenetic Meta-Patterns: A framework proposal for Performance-Generated Architectures
}

\author{
Pablo Salvador Banda \\ Non Consensual Architectural Practice \\ Pontificia Universidad Católica de Chile \\ pablo.banda.p@hotmail.com
}

\author{
Juan Eduardo Subercaseaux \\ SAW Architectural Technologies \\ Pontificia Universidad Católica de Chile \\ juane.saw@gmail.com
}

\begin{abstract}
Architecture and Technology's relationship nowadays has arrived to multi-disciplinary practices; they are growing exponentially while keeping away from to architectural discourse, are remarkable for its expressive power and the ability to solve complex problems. This opens the possibility for the generation of the organic, a path discarded by the Modern Movement in the past.Our approach explores three active premises called Morphogenetic Meta-Patterns: discrete processes (systemic guidelines) for the development of performance-based generative systems. These processes arise from Generative Design and their associated paradigms in the creation of a Framework between architecture and related disciplines.
\end{abstract}

Keywords: Design of parametric systems; Part-to-whole debate; Generative design.

\section{Introducción}

La práctica actual de la arquitectura digital está masificada y alcanzando un grado de madures tal, que le ha permitido dejar atrás la fascinación por lo nuevo y computacionalmente posible, para centrarse en llevar la arquitectura digital hacia una zona multidisciplinar y certera. Nuestro enfoque indaga en tres premisas activas que forman los llamados Meta-Patrones Morfogenéticos (desde ahora MM's): lineamientos sistémicos para el desarrollo de sistemas generativos basados en desempeño. Estos lineamientos invocan ideas dentro del Diseño Generativo y paradigmas implicados para la creación de un Framework de trabajo entre la arquitectura y las otras disciplinas.

Los MM se encargan de conducir un estudio de aspectos relevantes para el diseño, implícitos en la práctica arquitectónica computacional, estos son: Orientación a las versiones en arquitectura (MM01), de y re-composición de objetos y gradientes de desempeño (MM02), arquitectura como gestión y condicionamiento de complejidades (MM03). En su conjunto, estos Meta-Patrones forman un Modelo Hipotético, una composición propuesta como metodología pre-arquitectónica con potencial de extender la frontera disciplinar en un terreno multidisciplinario y fértil.

\section{Patrones}

\section{Patrones y meta-patrones}

Los patrones son soluciones genéricas para problemas bien descritos, claves para el aprendizaje y desarrollo de sistemas paramétricos (Woodbury 2011). El patrón trabaja en la transacción entre problema y solución, como diagrama constructivo generalizado (Alexander, 1968).

\section{Meta-patrón}

Un metapatrón está más arriba que los patrones, de alguna manera administrándolos. El significado de metapatrón es distinto en distintas ramas disciplinares:

Biología (1 definición): Meta-patrones como formas y organizaciones distinguibles en las cuales las formaciones naturales convergen independiente de su escala (Volk et al., 2007). El metapatrón biológico destaca las formas y organizaciones, lo que es afín tanto a las tipologías de la arquitectura tradicional, como al modo de concebirla (bosquejos, formas para la concepción de un objeto), en ese sentido ese enfoque de metapatrón biológico está bastante desarrollado en la disciplina.

Informática: Principio de construcción de un framework, entendiendo como framework un grupo de clases diseñada para trabajar en conjunto en la creación de un sistema (Pree, 1997).

El Metapatrón Morfogenético trabaja con la segunda definición por parte de la informática: a modo de framework abstracto el diseño de procesos de los sistemas generativos. La definición de Meta-Patrones Morfogenéticos necesariamente debe trabajar desde las siguientes distinciones: 
- Arquitectura y Diseño en general corresponden a actividades inventivas y propositivas.

- El dominio del uso de formas y objetos en general está ampliamente explorado.

Esta segunda distinción plantea una segunda línea de indagación, alejada del objeto e interesada en el desarrollo de procesos.

\section{Metapatrones y Ejercicios}

\section{MM01 - Versionaje: Espacio Explícito Pre-definido}

"Versioning is at the core of the digital form itself; its signature and its authenticity derive from the parameterized repetition which gives computer-generated design its characteristic combination of tightly disciplined structure and formal variability. It's not just the new calculuspowered curvaceousness, which is characteristic of a digitally informed age; it is also a disciplined groundwork of order that underpins the whole operation -- the rhythm of a powerful Turing Machine that drives the versioning at the heart of the digital aesthetic." Ingeborg Rocker.

La evaluación de soluciones de diseño en un modelo paramétrico corresponde a un ejercicio de su invariancia (Cache, 2011): todas la versiones de diseño pre-existen en un modelo paramétrico desde que trabajan con variables declaradas en una expresión formal. En ese sentido y según Robert Woodbury (2006) el total de las posibilidades del modelo paramétrico crea un espacio implícito de diseño. A su vez, la visita y recaudación de información del espacio paramétrico puede generar un espacio explícito de diseño.

\section{Diseño de Base de Datos}

La base de datos como diseño de información arquitectónica, plantea dentro de su ordenamiento y límites la conjugación de posibilidades desde los modelos paramétricos. Eventualmente se podría pasar de una base de datos de instanciación extensiva (basada en producto cartesiano de variables) a una de instanciación intensiva, introduciendo el algoritmo genético como motor de búsqueda.

Una buena estrategia para evitar la re-simulación para cada situación específica en un contexto (también específico) es diseñar un ejercicio previo, que incluya todas las posibles conjugaciones de variables, así estos resultados se podrían recaudar y ser propagados al reconocer los parámetros de entrada de la situación específica.

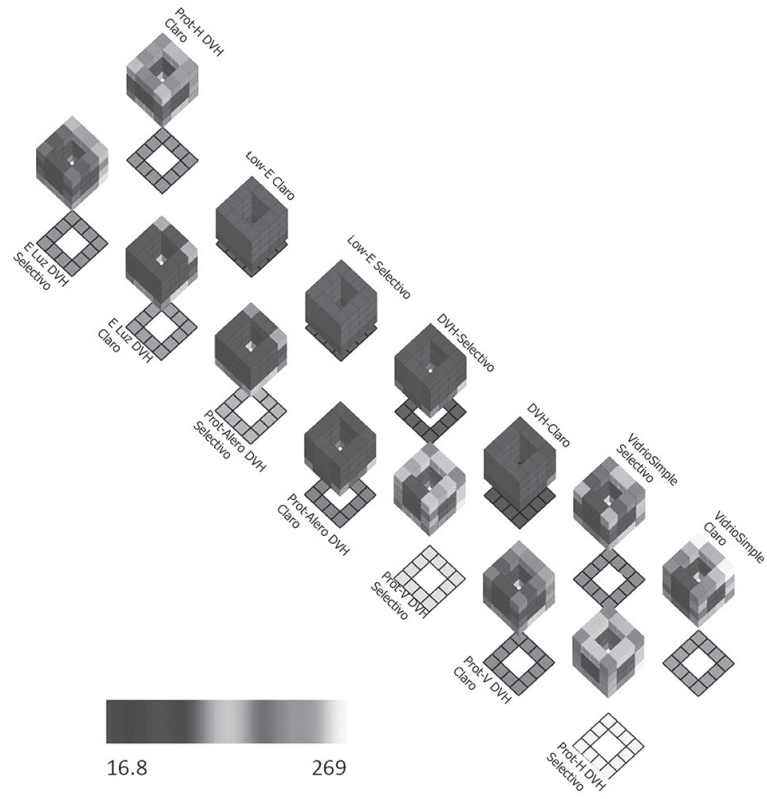

Figura 1: Edificios y mapeo de demandas de calefacción. Confección propia.

\section{Ejercicio: Análisis de sensibilidad ${ }^{1 .}$}

Este ejercicio pretende montar y evaluar una base de datos del comportamiento energético según orientaciones, porcentajes de acristalamiento, tipos de acristalamiento y tipos de protecciones solares (Figura 01). El edificio y el cuerpo de expresiones que lo definen son tomados como un modelo paramétrico; los resultados (y su orden) permiten un análisis de sensibilidad y a su vez una base de datos.

En ingeniería el análisis de sensibilidad corresponde a la evaluación de la influencia que tienen las variables sobre un sistema. Para realizar este proceso se debe establecer una manera ordenada de conjugar los parámetros tal que contribuya a revelar dicha influencia. A su vez, para crear un espacio explícito predefinido esta conjugación podrá llevarse a cabo mediante el producto cartesiano (Woodbury, 2010) de las variables, una vez fijados los intervalos relevantes.

En este trabajo se utilizaron los coeficientes de rendimiento y las demandas de frío y calor para todas las soluciones, desde donde se armó una solución miscelánea como un óptimo (Figura 02). 

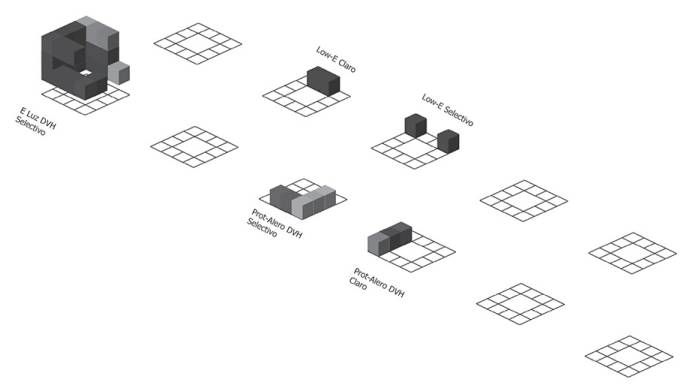

N
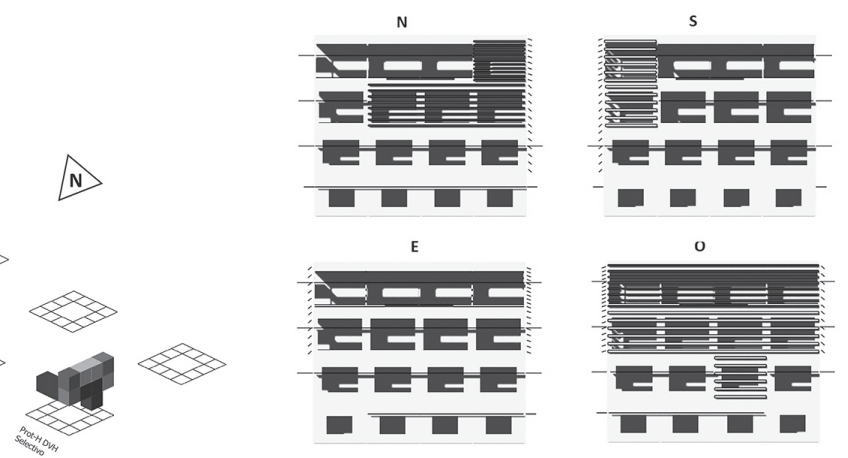

Figura 2: Deducción de Óptimo y su lugar en la Base de Datos, Confección propia.

\section{MM02 - Composición Intensiva}

\section{Composición y recurrencia}

Una obra de arquitectura está constituida generalmente por componentes, pudiendo estos estar formados de otros subcomponentes con fronteras en la mayoría de los casos bien definidas. Según Robert Aish el ámbito de la composición ha sido establecido como una de las aptitudes fundamentales para desarrollar una práctica paramétrica, siendo la utilización de los medios digitales una ventaja en cuanto a poder desarrollar y testear rápidamente estrategias de composición (Aish, 2009).

Según la Teoría General de Sistemas, es posible concebir las propiedades y modos de sistemas como independientes de escala: desde física hasta biología es un hecho la recurrencia de cualidades de los sistemas como relaciones entre partes como expresiones matemáticas (Bertanlaffy, 1950).

\section{Límites y diferenciación.}

El límite de un sistema con el contexto se establece como un modo de relación y respuesta al contexto mediante el desarrollo de complejidad interna.(Luhmann, 1990)
A nosotros nos interesa la visión independiente de escala de creación de límites y sistemas, como un proceso inminente de especificación arquitectónica. La denominación de contexto correspondería entonces a la declaración de una condición de borde para desarrollar sistemas desde unidades y transferir información desde la unidad base a sus partes. Esta información corresponde a una gradiente de desempeño a escala de objeto a traducir en gradientes para las partes.

\section{Ejercicio: Sliced forces $\mathbf{4}$ fab}

Este ejercicio examina el proceso de descomponer una figura "total" en un sistema de piezas, importa acá el re-leer la información tanto geométrica como de desempeños (Figura 03), entonces:

1 La orientación a la manufactura plantea la descomposición de una masa en elementos bidimensionales producibles por tecnología CNC $2 \mathrm{D}$ y 2 $1 / 2$ D.

2 La gradiente de esfuerzos desde la masa a las secciones es llevada desde el 3D al 2D, como un set de cargas locales. Un conjunto de otras fuerzas componen un nuevo contexto.
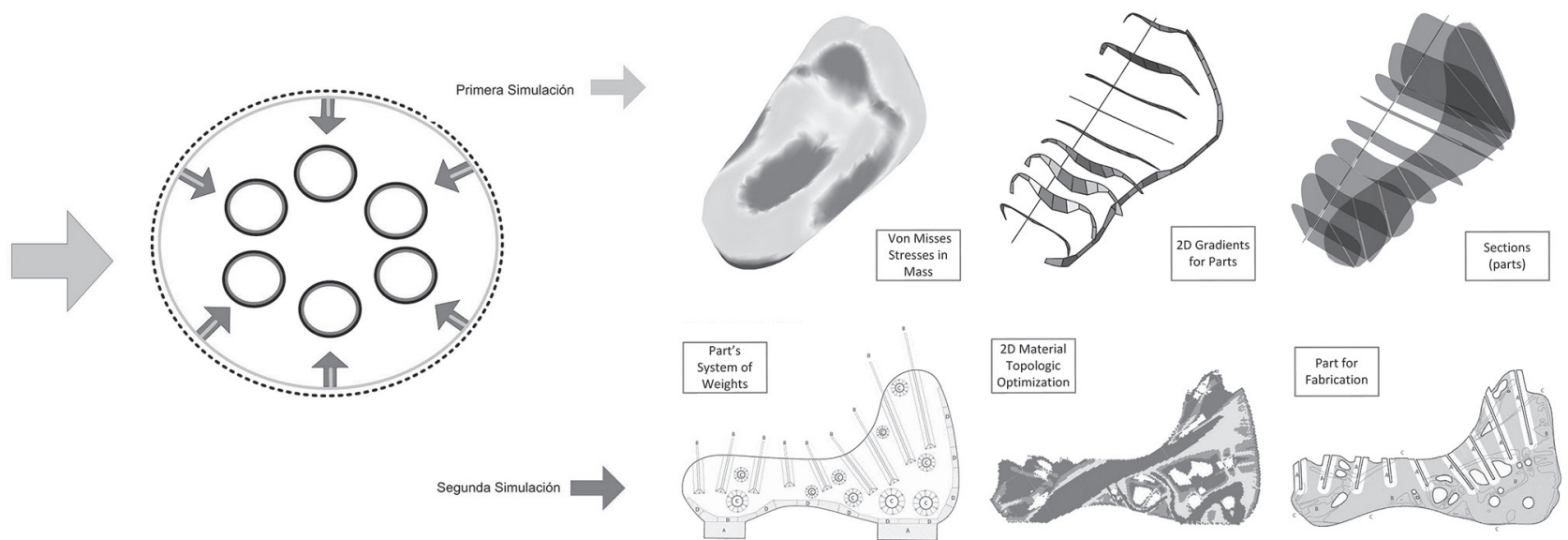

Figura 3: Relacion objeto-partes entre piezas y gradientes contextuales, Confección propia.

La importancia de este MM02 es la capacidad de establecer una mirada trans-escalar, de manera de poder tener dominio creativo y coherencia de la forma y su información a través de las diferentes escalas. 


\section{MM03 - Gestión Sistémica}

"This approach seems to provide a nice paradigm for architecture as the emergent outcome of a whole lot of interconnected feedback loops, which replace top-down geometry and the reductionist tradition, with dynamic relations and emergent outcomes not defined in the underlying model. At the same time, this leads to an alternative authorial voice - the illustrated consensus of the swarm for instance - to replace the search for a 'designer'" (Coates, 2010, p. 1).

\section{Perspectiva}

El éxodo del clásico esquema de arquitecto autor sugiere un cambio de perspectiva (Ruwan et al. 2010), bajando quizás desde donde la arquitectura es un "juego de los volúmenes bajo la luz" (tercera persona, desde afuera) hacia otra donde se trabaje con composiciones de elementos, y relaciones de vecindad individualizadas para cada uno de ellos (primera persona, desde adentro).

La perspectiva como punto de vista de la modelación de sistemas paramétricos produce relaciones y restricciones constitutivas del diseño (Ruwan et al. 2010): Los alcances son diversos entre las distintas instancias de diseño generativo ${ }^{2}$.

\section{Alcances desde la declaración de una gestión}

El meta-patrón plantea a priori la imagen del prototipo: establece concienzudamente la perspectiva de los elementos constitutivos y circunscribe el nivel de relaciones posibles; esto ejercitando la capacidad de embeber e inferir comportamientos en las soluciones emergentes de diseños generativos, esta capacidad plantearía efectivamente si un acto de diseño generativo es diseño (Stiny, 2009). El régimen de Gestión puede admitir flexibilidades, de manera de dejar terreno para resultados emergentes, o de lo contrario puede hacer padecer a la materia y la forma en campos infértiles de monotonía y sin sentido.

\section{Ejercicio: Swarm-based cell propagation}

Este trabajo explora la Gestión de la interacción (diseño de relaciones y retro-alimentación) en una estrategia desde la base hacia arriba (bottom-up). La meta de este trabajo era la capacidad de gestión de indicadores de desempeño para generar órdenes mayores de organización.

El comportamiento de estas células está inspirado en los Boids de Craig Reynolds, aunque está pensado para la convergencia hacia máximos en gradientes desde lugares arbitrarios. Los insumos principales en el contexto fueron las pendientes en el caso Bidimensional y Valores de Radiación en el caso 3D. Para ambos casos los comportamientos de dirección (steering behaviors) fueron

1 Una célula se mueve en cada paso hacia las locaciones con el valor máximo acercándose hacia sus vecinos

2 Si la celda se intersecta con sus vecinos, la magnitud de movimiento será dismunuida llegando hasta cero según el porcentaje de su superficie que está cubierta por esa intersección

Como se nota en los puntos 1 y 2 las relaciones entre células están establecidas en una especie de atracción global (la que conlleva a una atracción indirecta o agrupación de céldas hacia área específicas de la gradiente), y una relación de viscosidad entre las células (Figura 04).

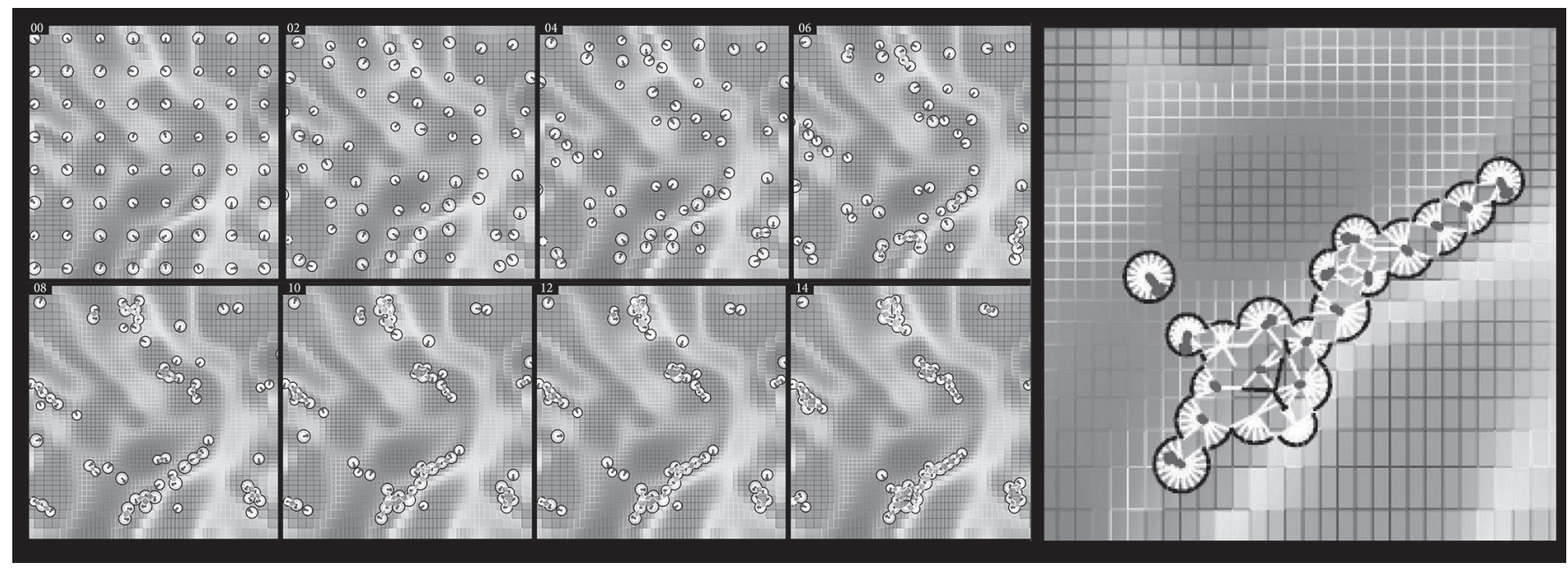

Figura 04: "Sistema emergente de células bidimensionales: rames de animación". Confección propia.

\section{Conclusión}

Desde este nuevo enfoque presentado pensamos se podrá retrotraer a la disciplina de la arquitectura la práctica digital-generativa. Ejemplos y literatura fundante ha sido provista por este escrito, como trabajo en el futuro cercano se realizará un sistema generativo incluyendo estos tres Metapatrones. 


\section{Framework y modelo recursivo}

La concepción de sistema como posible poseedor de $n$ dimensiones viene a coordinar los meta-patrones como encargados generar sistemas entre partes e introducirse en la parte para producir otros sistemas. Este ciclo es producido por un prototipo, o el código puntual que está aterrizando las ideas. De manera ilustrativa, los MM's en el prototipo deberían generar arquitectura en una recursión de los siguientes pasos:

Un ambiente de elementos primitivos a diferenciar: Su sistema de relaciones está producido bajo un metapatrón de gestión (MM03).

1 Se ejecuta la diferenciación accediendo a una base de datos de posibles casos (MM01)

2 Desde este estado actual diferenciado se ejecuta una De-Composición Intensiva (MM02) que re-instancia un nuevo sistema de relaciones influído metapatrón de gestión (MM03).

\section{Implicancias}

El manejo de procesos generativos teniendo en cuenta los MM's presentados, se plantea como el ejercicio de poder organizacional con perspectivas de mandato, logrando el cambio del esquema de un arquitecto autor por uno de un arquitecto como desarrollador de políticas.

\section{Agradecimientos}

Gracias a los docentes de la Universidad Católica de Chile cuyas cátedras se instanciaron los ejercicios acá discutidos: Waldo Bustamante, Arturo Lyon y Rodrigo Culagovsky.

\section{Referencias}

Aish, R.(2005) From Intuition to Precision, Conferencia eCAADe 23, 10-14.

Alexander, C. (1968). "A pattern language: towns, buildings, construction". New York: Oxford University Press.

Alexander, C. (2005) Harmony-Seeking Computations: a Science of NonClassical Dynamics based on the Progressive Evolution of the Larger Whole. The Grand Challenge in Non-Classical Computation (workshop internacional, 2005).
Bertanlaffy, L. von. (1950). An Outline of General System Theory. The British Journal for the Philosophy of Science, 2(2), 134-165.

Bustamante, W., Encina, F., Parámetros de Diseño y Desempeño en Edificios de Clima Mediterráneo. ARQ 82, 16-19.

Cache, B.(2011) Towards a Non-Standard Mode of Production. Projectiles (Architectural Words), Londres: Architectural Association.

Coates, P. (2010) Programming Architecture, Nueva York: Routledge.

Luhmann, N. (1998) Sistemas Sociales: Lineamientos para una teoría general. Mexico: Anthropos.

Pree, W. (1997) Design Patterns for Object-Oriented Software Development. Austria: Kepler University

Rocker, I. (2008) Versioning: Architecture as Serie. Conferencia Critical Digital (1), Cambridge: Harvard 157-170.

Ruwan, F. Drogemuller, R. Dilys Salim, F., Burry, J. (2010) Patterns, heuristics for architectural design support: making use of evolutionary modelling in design. Conferencia CAADRIA 15, 283-292.

Stiny, G. (2009) When is Generative Design Design?, Presentación Oral, Conferencia eCAADe (27), Estanbul: Turkía, 16-19.

Volk, T., Bloom, J., Richards, J. (2007) "Toward a science of metapatterns: building upon Bateson's foundation", Revista Kybernetes, 36(7/8), 1070-1080.

Wisse, P. (2001). Metapattern: context and time in information models, Addison-Wesley.

Woodbury, R. (2010) Elements of Parametric Design. Vancouver: Routledge.

Woodbury, R., Burrow, A. (2006) Whither Design Space?. Artificial Intelligence for Engineering Design, Analysis, and Manufacturing, 20(2), pp 63-82.

\section{Notas}

1. Trabajo realizado por Pablo Banda en el contexto de la cátedra "Evaluación Ambiental de Edificios" encabezada por Waldo Bustamante el 1er Semestre del 2013. Este ejercicio fue basado en un a investigación antes publicada como publicado como: Parámetros de Diseño y Desempeño en Edificios de Clima Mediterráneo.

2. Trabajo realizado por Delia Farrell y Pablo Banda en el contexto de la cátedra Tecnologías Aplicadas al Proyecto de Arquitectura, encabezado por Arturo Lyon el 1er Semestre del 2013.

3. Trabajo realizado por Pablo Banda en el contexto del laboratorio de arquitectura MARQLAB, encabezado por Rodrigo Culagovsky el 2do Semestre del 2012. 